\title{
Reappraisal of Tele-Surgery in the Era of High- Speed, High-Bandwidth, Secure Communications: Evaluation of Surgical Performance in Local/Remote Environments
}

\section{Yoshiya Takahashi}

Department of Gastroenterological Surgery, Hirosaki University Graduate School of Medicine, Hirosaki, Japan Committee for Promotion of Remote Surgery Implementation, Japan Surgical Society, Tokyo Kenichi Hakamada ( $\square$ hakamada@hirosaki-u.ac.jp)

Department of Gastroenterological Surgery, Hirosaki University Graduate School of Medicine, Hirosaki, Japan Committee for Promotion of Remote Surgery Implementation, Japan Surgical Society, Tokyo Hajime Morohashi

Department of Gastroenterological Surgery, Hirosaki University Graduate School of Medicine, Hirosaki, Japan Committee for Promotion of Remote Surgery Implementation, Japan Surgical Society, Tokyo Harue Akasaka

Department of Gastroenterological Surgery, Hirosaki University Graduate School of Medicine, Hirosaki, Japan Committee for Promotion of Remote Surgery Implementation, Japan Surgical Society, Tokyo

\section{Yuma Ebihara}

Department of Gastroenterological Surgery II, Hokkaido University Faculty of Medicine, Sapporo

\section{Eiji Oki}

Department of Surgery and Science, Kyushu University, Fukuoka

\section{Satoshi Hirano}

Department of Gastroenterological Surgery II, Hokkaido University Faculty of Medicine, Sapporo

\section{Masaki Mori}

Tokai University School of Medicine, Isehara

\section{Research Article}

Keywords: Robotic surgery, telesurgery, glass-to-glass time, communication delay

Posted Date: February 22nd, 2022

DOI: https://doi.org/10.21203/rs.3.rs-1365089/v1

License: (c) (i) This work is licensed under a Creative Commons Attribution 4.0 International License. Read Full License 
Page 2/15 


\section{Abstract}

Communication and video transmission delays negatively affect telerobotic surgery. Since latency varies by communication environment and robot, to realize remote surgery, both must perform well. This study aims to examine the feasibility of telerobotic surgery by validating the communication environment and local/remote robot operation, using secure commercial lines and newly developed robots.

Hirosaki University and Mutsu General Hospital, $150 \mathrm{~km}$ apart, were connected via Medicaroid's surgical robot. Ten surgeons performed a simple task remotely using information encoding and decoding. The required bandwidth, delay time, task completion time, number of errors, and image quality were evaluated. Next, 11 surgeons performed a complex task using gallbladder and intestinal models in local/remote environments; round trip time (RTT), packet loss, time to completion, operator fatigue, operability, and image were observed locally and remotely.

Image quality was not degraded so as to affect remote robot operation. Median RTT was 4 msec (2-12), and added delay was $29 \mathrm{msec}$. There was no significant difference in accuracy or number of errors for cholecystectomy, intestinal suturing, completion time, surgeon fatigue, or image evaluation.

The fact that remote surgery succeeded as well as local surgery showed that this system has the necessary elemental technology for widespread social implementation.

\section{Introduction}

Currently, surgical assist robots are deployed in a very large number of hospitals worldwide, and they are also becoming popular in Japan. In addition, there is a great demand for minimally invasive surgery, such as robotic surgery ${ }^{1}$. Furthermore, there are high expectations for telerobotic surgery using commercial lines since optical fiber and $5 \mathrm{G}$ networks are available nationwide ${ }^{2,3}$. Telerobotic surgery has been studied since the 1970s, and the world's first tele-laparoscopic cholecystectomy was performed between the US and France in $2001^{4}$. This was followed by a number of remote surgeries in Canada ${ }^{5}$, all of which were reported to be successful. However, many issues exist, such as the economics of dedicated communication lines, communication security, and communication delays, so widespread use has not progressed since those early experiments. In recent years, viable communication networks have been developed, and the issues of communication delay and security are being resolved. In addition, the development of new surgical robots has progressed, and various models are now available on the market

6 . We conducted a demonstration of telerobotic surgery using a commercial line as a preliminary study in February 2021, and reported that it was possible to build a communication environment conducive to remote surgery ${ }^{7}$. However, since the communication delay and the video transmission information delay vary depending on the communication environment to be constructed and the robot to be used. Therefore, it is necessary to verify communication environments and robots used in various combinations. 
The purpose of this study is to examine the communication environment and robot operability under local and remote environments using Japanese commercial lines and new robots already available on the market, and to examine the possibility of social implementation of tele-surgery.

\section{Material And Methods}

\section{Communication Environment}

Hirosaki University Hospital (Hirosaki City, Aomori, Japan) and Mutsu General Hospital (Mutsu City, Aomori, Japan), $150 \mathrm{~km}$ apart, were connected by fiber optic network provided by Nippon Telegraph and Telephone East Corporation. The guaranteed bandwidth of the fiber optic network is $200 \mathrm{Mbps}$, and an IPVPN (Internet Protocol-Virtual Private Network) is constructed. A guaranteed line is one in which the bandwidth can be selected according to the subscriber's purpose, and comes with a service quality guarantee and 24-hour/365-day support service as standard. The data volume of the transmitted image information was limited to $120 \mathrm{Mbps}$ by encoder and decoder. We evaluated the roundtrip time (RTT) of the network line, the delay time of the encoder and decoder, and the packet loss of the image during the telerobotic tasks.

\section{Surgery Robot}

The surgical robot was hinotori ${ }^{\mathrm{T}}$, a surgical assist robot developed by Medicaroid Corporation. The hinotorit⿳ $^{\text {TM }}$ consists of three units: the operation unit, the surgeon's cockpit, and the vision unit. The surgeon's cockpit is operated by a hand controller while viewing a closed 3D monitor.

\section{Simple Tasks}

The subjects were 10 surgeons with sufficient surgical experience, but mixed experience in robotic surgery who performed the relocation of a triangular prism remotely. This was a two-handed task. Six Triangles ${ }^{\text {TM }}$

8 were placed at designated locations on a peg board sitting on a table, which mechanically rocked to mimic respiratory fluctuation (TRP-BHT1, by KOTOBUKI medical Inc.). For the background, we used a cloth printed with intra-abdominal images of the upper abdomen, mainly the gallbladder, to simulate a real intraperitoneal environment (Fig.1). In follow-up tasks, six triangular prisms were inserted into the rods on the left side, which were moved to the right side, or, conversely, six triangular prisms on the left side were moved to the right side. If the operator could not get hold of, dropped, or incorrectly inserted the Triangles, that attempt was counted as an Error.

\section{Complex Tasks}

The subjects were 11 surgeons with extensive surgical experience. Each of them performed two tasks, a cholecystectomy and intestinal suturing, under both non-remote and remote conditions.

\section{(1) Cholecystectomy}


We used a gallbladder model manufactured by FASOTEC Co., Ltd. The right hand was used as a monopolar arm and the left hand as a bipolar arm that was dissecting using an electrocautery. After identifying the cystic duct and the cystic artery, they were ligated proximally and distally, once each, with 2-0 silk thread and separated. The gallbladder was then removed from the gallbladder bed to complete the task. The silk thread was provided and collected by the assistant. An evaluation method reported by Sharker et al. was modified to evaluate the accuracy, number of errors, and time required ${ }^{9}$. The number of errors was scored on a scale of 0-6 for each ligation procedure. Accuracy was scored on a scale of 0-6, with $\mathrm{YES}=1$ and $\mathrm{NO}=0$ for the six items.

\section{(2) Intestinal suturing}

For intestinal suturing, we used an intestinal model made by AS ONE Corporation. Five suture sites were marked at $5 \mathrm{~mm}$ intervals, and three single ligations were performed in one suture (Fig.2). After ligation, the sutures were detached by an assistant. The time from the start of suturing to the fifth suture ligation and separation was measured and evaluated, and the accuracy and number of errors were evaluated by extracting the items that can be used for intestinal suturing from the evaluation method proposed by Goh et al ${ }^{10}$. Accuracy was evaluated on a 5-point scale (1-5), and the score was 5-25. The number of errors was evaluated using six items, and the cumulative value of the number of errors was used as the score.

\section{Evaluation of image quality}

\section{(1) Image Quality Score A}

In order to analyze, in detail, the type of image quality degradation caused by changes in the communication environment and the impact of that on the procedure, we created a rating scale for clarity, stereoscopic vision, completeness, continuity, and impact on the procedure (Supplementary table 1). The image quality scale is a 5-point scale, where a high score indicates that the image quality is not degraded and does not affect the procedure. The total score for each item was used for evaluation.

\section{(2) Image Quality Score B}

The Image Quality Score B was created based on the image evaluation method proposed by Mitsuhashi 11. Specifically, an image quality rating scale was created to evaluate the degradation of image quality due to changes in the communication environment and its effect on the procedure (Supplementary table 2). The image quality rating scale is a 5-point scale, where a high score indicates no degradation in image quality and no effect on the procedure. The median scores were compared.

\section{Evaluation of robot operability}

\section{(1) mSUS}

The usefulness of remote surgery was assessed by mSUS, a modified version of The System usability scale (SUS) proposed by Brook ${ }^{12}$. Nine items were each rated on a 5 -point scale, and the total score was 
calculated.

\section{(2) Robot Usability Score}

To evaluate the operability of the surgical robot, we modified the Robot Usability Score (Supplementary table 3$)^{13}$. Eight items were rated on five levels, and the total score was calculated.

\section{(3) PFS-12}

Subjective fatigue was assessed using the PFS-12. Four items (behavioral, affective, sensory, and cognitive) were rated on a scale of 0-10 and scored using the calculation method reported by Reeve et al 14 .

\section{Statistical analysis}

The software used was EZR ${ }^{15}$. The normality test was the kolmogorov-smirnov test, and when normality was not rejected, the paired t-test was used. When normality was rejected, the Wilcoxon signed rank sum test was used. Statistical significance was determined at $p<0.05$.

\section{IRB approval}

Because this research was not conducted on investigations that involve human subjects or laboratory animals, it was not necessary to obtain IRB approval.

\section{Results}

\section{Transmission delay time}

The RTT time was a median of 4 [2-12] msec. The delay between encoder and decoder was $25 \mathrm{msec}$ of the encoder-decoder processing time. Therefore, the remote delay time compared to the local environment was $29 \mathrm{msec}$.

\section{Video Signal Packet Loss And Surgical Field Images}

The measurement results of typical transmissions when the line bandwidth during the surgical task was set to $200 \mathrm{Mbps}$ are shown in the figure. The bandwidth usage for transmission signals was $145 \mathrm{Mbps}$, and no packet loss was observed.

\section{Evaluation Of Image Quality}

There was no significant difference in any of the five items of Image Quality Score A, and no difference in total score $(p=0.07)$. There was a significant difference in Image Quality Score B, $4.91[4-5]$ in local and 
$4.45[4-5]$ in remote $(p=0.04)($ Table 1$)$.

Table 1

Comparison of image quality between local and remote. Values are averages (ranges). * $\mathrm{p}<0.05$

\begin{tabular}{|llll|}
\hline & Local & Remote & p \\
\hline Image Quality Score A & $23.9(21-25)$ & $22.8(20-25)$ & 0.07 \\
\hline Image Quality Score B & $4.91(4-5)$ & $4.45(4-5)$ & $0.04^{\star}$ \\
\hline
\end{tabular}

\section{Evaluation Of Surgical Performance}

(1) Cholecystectomy task

The accuracy score was 5.0 [2.5-6] for local and 4.9 [3.0-6.0] for remote, and the number of errors was 1.0 [0-2.5] for local and 1.1 [0-3.5] for remote, with no significant difference $(p=0.82, p=0.74)$ (Table 2). The completion time was 1156.3 [973-1794] sec for local and 1247.3 [979-1380] sec for remote, with no significant difference $(p=0.70)$.

Table 2

Comparison of cholecystectomy task between local and remote. Values are averages (ranges).

\begin{tabular}{|llll|}
\hline & Local & Remote & p \\
\hline Accuracy (score) & $5.0(2.5-6)$ & $4.9(3.0-6.0)$ & 0.82 \\
\hline Errors (score) & $1.0(0-2.5)$ & $1.1(0-3.5)$ & 0.74 \\
\hline Total time (sec) & $1156.3(973-1794)$ & $1247.3(979-1380)$ & 0.70 \\
\hline
\end{tabular}

(2) Intestinal suturing task

The accuracy score was 22.8 [19.5-25] for local and 22.5 [18.5-25] for remote, and the number of errors was $1.4[0-4]$ for local and 1.8 [0-5.5] for remote, with no significant difference $(p=0.43, p=0.29)$. The completion time was 599.1 [350-846] sec for local and 610.3 [327-831] sec for remote, with no significant difference $(p=0.73)$ (Table 3$)$. 
Table 3

Comparison of intestinal suturing task between local and remote. Values are averages (ranges).

\begin{tabular}{|llll|}
\hline & Local & Remote & P \\
\hline Accuracy (score) & $22.8(19.5-25)$ & $22.5(18.5-25)$ & 0.43 \\
\hline Errors (count) & $1.4(0-4)$ & $1.8(0-5.5)$ & 0.29 \\
\hline Total time (sec) & $599.1(350-846)$ & $610.3(327-831)$ & 0.73 \\
\hline
\end{tabular}

\section{Evaluation Of Robot Operability And Surgeon Fatigue}

The total mSUS score was 35.5 [28-44] for local and 34.6 [29-44] for remote, and no significant difference was observed $(p=0.09)$ (Table 4). The total Robot Usability Score was 34.6 [26-40] for local and 33.5 [27-40] for remote $(p=0.30)$ (Table 5). No significant difference was found in any subscale. There was no significant difference in total score before and after the procedure as assessed by PFS-12: 27.0 to $30.9(p=0.15)$ for local and 31.5 to $27.2(p=0.53)$ for remote. There was a significant difference in the "behavior" subscale from 2.2 to $2.9(p=0.01)$ in the local group (Table $6-1)$. No difference was found in the total score or in the degree of change for any subscale (Table $6-2)$.

Table 4

Evaluation of usefulness of remote surgery. Values are averages (ranges).

\begin{tabular}{|llll|}
\hline & Local & Remote & p \\
\hline mSUS & $35.5(28-44)$ & $34.6(29-44)$ & 0.09 \\
\hline
\end{tabular}


Table 5

Evaluation of robot operability. Values are averages (ranges).

\begin{tabular}{|llll|}
\hline & Local & Remote & p \\
\hline Physical Comfort & $4.1(3-5)$ & $3.9(3-5)$ & 0.42 \\
\hline Hand Controls & $4.4(3-5)$ & $4.2(3-5)$ & 0.42 \\
\hline Foot Controls & $4.0(2-5)$ & $3.8(2-5)$ & 0.59 \\
\hline 3D Vision & $4.4(4-5)$ & $3.9(2-5)$ & 0.09 \\
\hline Annoyed or Stressed & $4.2(2-5)$ & $4.1(3-5)$ & 0.77 \\
\hline Smooth & $4.6(4-5)$ & $4.6(4-5)$ & 1.00 \\
\hline Satisfaction & $4.5(4-5)$ & $4.5(3-5)$ & 0.77 \\
\hline Actuality & $4.5(4-5)$ & $4.4(4-5)$ & 0.35 \\
\hline Total & $34.6(26-40)$ & $33.5(27-40)$ & 0.30 \\
\hline
\end{tabular}

Table 6

-1 Evaluation of surgeon fatigue. Values are averages (ranges). ${ }^{*} p<0.05$

\begin{tabular}{|c|c|c|c|c|c|c|}
\hline & Local & & & Remote & & \\
\hline & before & after & $p$ & before & after & $p$ \\
\hline All score & $\begin{array}{l}27.0(0.0- \\
64.0)\end{array}$ & $\begin{array}{l}30.9(2.0- \\
63.0)\end{array}$ & 0.15 & $\begin{array}{l}31.5(0.0- \\
82.0)\end{array}$ & $\begin{array}{l}27.2(0.0- \\
63.0)\end{array}$ & 0.53 \\
\hline Behavioral & $2.2(0.0-6.0)$ & $2.9(0.7-6.0)$ & $0.01 *$ & $2.4(0.0-8.3)$ & $2.7(0.0-7.3)$ & 0.27 \\
\hline Affective & $2.6(0.0-5.3)$ & $2.9(0.0-6.7)$ & 0.20 & $3.0(0.0-7.0)$ & $2.5(0.0-6.0)$ & 0.19 \\
\hline Sensorial & $2.5(0.0-5.0)$ & $2.4(0.0-5.0)$ & 0.88 & $2.9(0.0-6.7)$ & $2.2(0.0-5.0)$ & 0.27 \\
\hline Cognitive & $1.7(0.0-5.0)$ & $2.0(0.0-5.0)$ & 0.12 & $2.2(0.0-5.3)$ & $1.7(0.0-5.0)$ & 0.40 \\
\hline
\end{tabular}

Table 6

- 2 Degree of change in fatigue before and after the task. Values are averages (ranges).

\begin{tabular}{|llll|}
\hline PFS-12 & $\Delta$ Local & $\Delta$ Remote & $\mathbf{p}$ \\
\hline All score & $3.90(-12-12)$ & $-4.36(-46-13)$ & 0.20 \\
\hline Behavioral & $0.75(-0.3-2.3)$ & $0.26(-4.7-3)$ & 0.40 \\
Affective & $0.37(-3-1.7)$ & $-0.48(-2.7-1)$ & 0.08 \\
\hline Sensorial & $-0.12(-2-1.3)$ & $-0.75(-4.7-0.7)$ & 0.61 \\
\hline Cognitive & $0.31(-0.7-1)$ & $-0.47(-3.3-1)$ & 0.20 \\
\hline
\end{tabular}




\section{Discussion}

As a result, the delay time added to the normal local environment was $29 \mathrm{msec}$; no packet loss was observed, and no degradation of image quality that would affect robot operation due to information encoding and decoding was apparent. We also demonstrated that robot operation in the local environment can be reproduced at the same performance level in a remote environment. The results of this study were elucidated in a complex task similar to a real clinical situation. Using an artificial organ model on a table with respiratory-like movement, robotic surgery using commercial lines and Japanese robots has the elemental technology necessary for social implementation of remote surgery.

In general, telerobotic surgery suffers from problems of communication and transmission delays that interfere with operations compared to what is expected with local surgery ${ }^{16}$. There have been many reports on the extent to which communication delay is acceptable; Christopher et al. reported on the validation of pyeloplasty in pigs using telerobotic surgery, and found that the average delay was $66.1 \pm$ $1.5 \mathrm{msec}$, and no operability or visual impact on the surgeon was observed ${ }^{17}$. It is generally believed that a delay time of 100 msec or more affects operability and makes surgery difficult ${ }^{18,19}$. In this study, the added delay in the remote environment was $29 \mathrm{msec}$, which was the sum of the communication delay due to the line and the delay due to the information processing in the encoder/decoder, compared to the normal local environment. It was an acceptable delay time that did not interfere with general surgery.

As for the evaluation of image quality, although no packet loss or fluctuation occurred in the communication environment, some subjects felt that the image quality was degraded, but it did not affect the surgical procedure in the remote environment. Image quality information is mainly composed of three elements: resolution, color, and frame rate. In order to communicate image quality information, the information must be compressed by the encoder and decompressed by the decoder, and how and which of these elements are compressed and decompressed depends on the capabilities of the codec and the bitrate ${ }^{20}$. The bitrate is the amount of data that can be sent and received in one second. The higher the bit rate, the more beautiful the image can be delivered, but the larger the amount of data size to be transmitted, the more likely the communication will be interrupted. In the future, the key to the success of remote surgery will be how to process and transmit these three elements of image information without causing stress to the surgeon.

There was no difference in surgical performance between local and remote tasks. In order to realize telerobotic surgery, it is necessary to verify not just simple tasks, but more complex and clinical tasks, which are similar to actual surgery, such as suturing and ligation, or removing organs using an electric scalpel. The fact that there is no difference in operability in complex tasks using the artificial organ model in this study is considered to be a major achievement toward social implementation.

The mSUS was mainly used to evaluate the usefulness of the robot system, while the Robot Usability Score was used to subjectively evaluate robot operability. However, none of the differences were significant between local and remote, including the subscales. There have been few reports describing 
detailed evaluation criteria for manipulability of the robot arm and image distortion ${ }^{6}$. In this study, we made certain subjective and objective judgments using the various evaluation tables reported so far, and showed that there was no difference in the operability of the robot between remote and local tasks.

Previous studies using surgical robots have reported that surgical performance decreases when the workload is high ${ }^{21}$. In the present study, a significant difference was found in the subscales for the degree of fatigue, but this was thought to be due to the overlapping number of times the task was performed, rather than fatigue due to remote operation. There was no difference in the other items or in the overall degree of change, and no significant feeling of fatigue was observed. However, it has been reported that more than half of patients experience physical fatigue after a long robotic surgery ${ }^{22}$. In this study, the task was 20 minutes long at most, and it is necessary to examine how prolonged telerobotic surgery may affect the level of surgeon fatigue.

In Japan, a shortage of surgeons in rural areas exists due to the unequal distribution of doctors between urban and rural areas and the overall decrease in the number of surgeons. Telerobotic surgery is expected to provide an opportunity for young surgeons to work in rural areas, as it enables them to engage in local medical care in rural areas while providing surgical education and support for their supervisors at core hospitals ${ }^{1,23}$. In this environment, there are high expectations for telerobotic surgery, and early social implementation is required.

It would be ideal to have a communication line with a wide bandwidth that matches the required bandwidth of the robot. However, in general, communication lines using a wide bandwidth are extremely costly, and are likely to become a stumbling block to the social implementation and widespread use of telerobotic surgery. In the future, it was thought that innovations such as narrowing down the amount of information in the robot signal to match the communication bandwidth in consideration of economy would be required.

\section{Limitations}

Due to the limited verification time, the number of subjects was small, and it was not possible to randomly perform local and remote tasks. Only expensive leased lines with guaranteed bandwidth were used, and the verification did not take into account economical bandwidth alternatives or redundancy in case of communication interruptions. In addition, since only one type of communication bandwidth was set, there was no verification of behavior in situations where bandwidth was reduced.

\section{Conclusions}

The performance of remote surgery using hinotori ${ }^{\text {TM }}$ with full specifications over a commercial line was equivalent to that of the local environment. Therefore, remote surgery with hinotori ${ }^{\text {TM }}$ using a commercial line can be performed safely, indicating that we have the elemental technology necessary for social implementation of telerobotic surgery. 


\section{Declarations}

\section{Acknowledgements}

This study was done as part of the Advanced Telemedicine Network Research Project of the Japan Agency for Medical Research and Development (AMED), and we are grateful for the budget support. We would also like to express our deepest gratitude to Medicaroid Corporation, NTT East, Soliton Systems K.K., Hirosaki University, and Mutsu General Hospital for their cooperation in the experiments. We sincerely thank Shari Joy Berman for professionally editing the English draft of this manuscript.

\section{Disclosures}

This work was supported by a grant from the Japan Agency for Medical Research and Development (AMED), Grant Number JP21hs0122001h0002.

\section{Author Contributions}

Y.T. did the study design, analyzed the data, interpreted the results, and wrote the manuscript. K.H. provided oversight and guidance for the entire study, interpreted and offered analyses of the results. H.M. and H.A. verified the study design, took charge of the data collection, interpreted, and offered analyses of the results. Y.E., E.O., S.H., and M.M. supervised this research. All authors reviewed the final manuscript.

\section{Competing interests}

The authors declare no competing interests.

\section{Data availability}

Data is available from the corresponding author upon reasonable request.

\section{References}

1. Hakamada, K. \& Mori, M. The changing surgical scene: From the days of Billroth to the upcoming future of artificial intelligence and telerobotic surgery. Ann Gastroenterol Surg 5, 268-269, doi:10.1002/ags3.12466 (2021).

2. Tian, W. et al. Telerobotic Spinal Surgery Based on $5 \mathrm{G}$ Network: The First 12 Cases. Neurospine 17, 114-120, doi:10.14245/ns.1938454.227 (2020).

3. Xia, S. B. \& Lu, Q. S. Development status of telesurgery robotic system. Chin. J. Traumatol. 24, 144147, doi:10.1016/j.cjtee.2021.03.001 (2021).

4. Marescaux, J. et al. Transatlantic robot-assisted telesurgery. Nature 413, 379-380, doi:10.1038/35096636 (2001).

5. Anvari, M., McKinley, C. \& Stein, H. Establishment of the world's first telerobotic remote surgical service: for provision of advanced laparoscopic surgery in a rural community. Ann. Surg. 241, 460- 
464, doi:10.1097/01.sla.0000154456.69815.ee (2005).

6. Zheng, J. et al. 5G ultra-remote robot-assisted laparoscopic surgery in China. Surg. Endosc. 34, 5172-5180, doi:10.1007/s00464-020-07823-x (2020).

7. Morohashi H, Hakamada K, Kanno T, Kawashima K, Akasaka H, Ebihara Y, Oki E, Hirano S, Mori M. Social Implementation of Remote Surgery in Japan: A Field Experiment using a Newly Developed Surgical robot via Commercial Network. Surg. Today (2021).

8. Bernier, G. V. \& Sanchez, J. E. Surgical simulation: the value of individualization. Surg. Endosc. 30, 3191-3197, doi:10.1007/s00464-016-5021-8 (2016).

9. Sarker, S. K., Chang, A., Vincent, C. \& Darzi, A. W. Technical skills errors in laparoscopic cholecystectomy by expert surgeons. Surg. Endosc. 19, 832-835, doi:10.1007/s00464-004-9174-5 (2005).

10. Goh, A. C., Goldfarb, D. W., Sander, J. C., Miles, B. J. \& Dunkin, B. J. Global evaluative assessment of robotic skills: validation of a clinical assessment tool to measure robotic surgical skills. J. Urol. 187, 247-252, doi:10.1016/j.juro.2011.09.032 (2012).

11. Mitsuhashi.T. A short history of the subjective assessment method of picture quality(2). Journal of informatics for arts, Shobi University 15, 53-62 (2009).

12. Brooke, J. SUS: A quick and dirty usability scale. Usability evaluation in industry 189, 4-7 (1996).

13. Tanaka, A. et al. Robotic surgery simulation validity and usability comparative analysis. Surg. Endosc. 30, 3720-3729, doi:10.1007/s00464-015-4667-y (2016).

14. Reeve, B. B. et al. The Piper Fatigue Scale-12 (PFS-12): psychometric findings and item reduction in a cohort of breast cancer survivors. Breast Cancer Res. Treat. 136, 9-20, doi:10.1007/s10549-0122212-4 (2012).

15. Kanda, Y. Investigation of the freely available easy-to-use software 'EZR' for medical statistics. Bone Marrow Transplant. 48, 452-458, doi:10.1038/bmt.2012.244 (2013).

16. Rayman, R. et al. Robotic telesurgery: a real-world comparison of ground- and satellite-based internet performance. Int. J. Med. Robot. 3, 111-116, doi:10.1002/rcs.133 (2007).

17. Nguan, C., Miller, B., Patel, R., Luke, P. P. \& Schlachta, C. M. Pre-clinical remote telesurgery trial of a da Vinci telesurgery prototype. Int. J. Med. Robot. 4, 304-309, doi:10.1002/rcs.210 (2008).

18. Xu, S. et al. Determination of the latency effects on surgical performance and the acceptable latency levels in telesurgery using the dV-Trainer( $\left({ }^{\circ}\right)$ simulator. Surg. Endosc. 28, 2569-2576, doi:10.1007/s00464-014-3504-z (2014).

19. Kim, T., Zimmerman, P. M., Wade, M. J. \& Weiss, C. A., 3rd. The effect of delayed visual feedback on telerobotic surgery. Surg. Endosc. 19, 683-686, doi:10.1007/s00464-004-8926-6 (2005).

20. Sullivan, G. J., Ohm, J.-R., Han, W.-J. \& Wiegand, T. Overview of the high efficiency video coding (HEVC) standard. IEEE Transactions on circuits and systems for video technology 22, 1649-1668 (2012). 
21. Wu, C. et al. Eye-Tracking Metrics Predict Perceived Workload in Robotic Surgical Skills Training. Hum. Factors 62, 1365-1386, doi:10.1177/0018720819874544 (2020).

22. Lee, G. I., Lee, M. R., Green, I., Allaf, M. \& Marohn, M. R. Surgeons' physical discomfort and symptoms during robotic surgery: a comprehensive ergonomic survey study. Surg. Endosc. 31, 1697-1706, doi:10.1007/s00464-016-5160-y (2017).

23. Mohan, A., Wara, U. U., Arshad Shaikh, M. T., Rahman, R. M. \& Zaidi, Z. A. Telesurgery and Robotics: An Improved and Efficient Era. Cureus 13, e14124, doi:10.7759/cureus.14124 (2021).

\section{Figures}

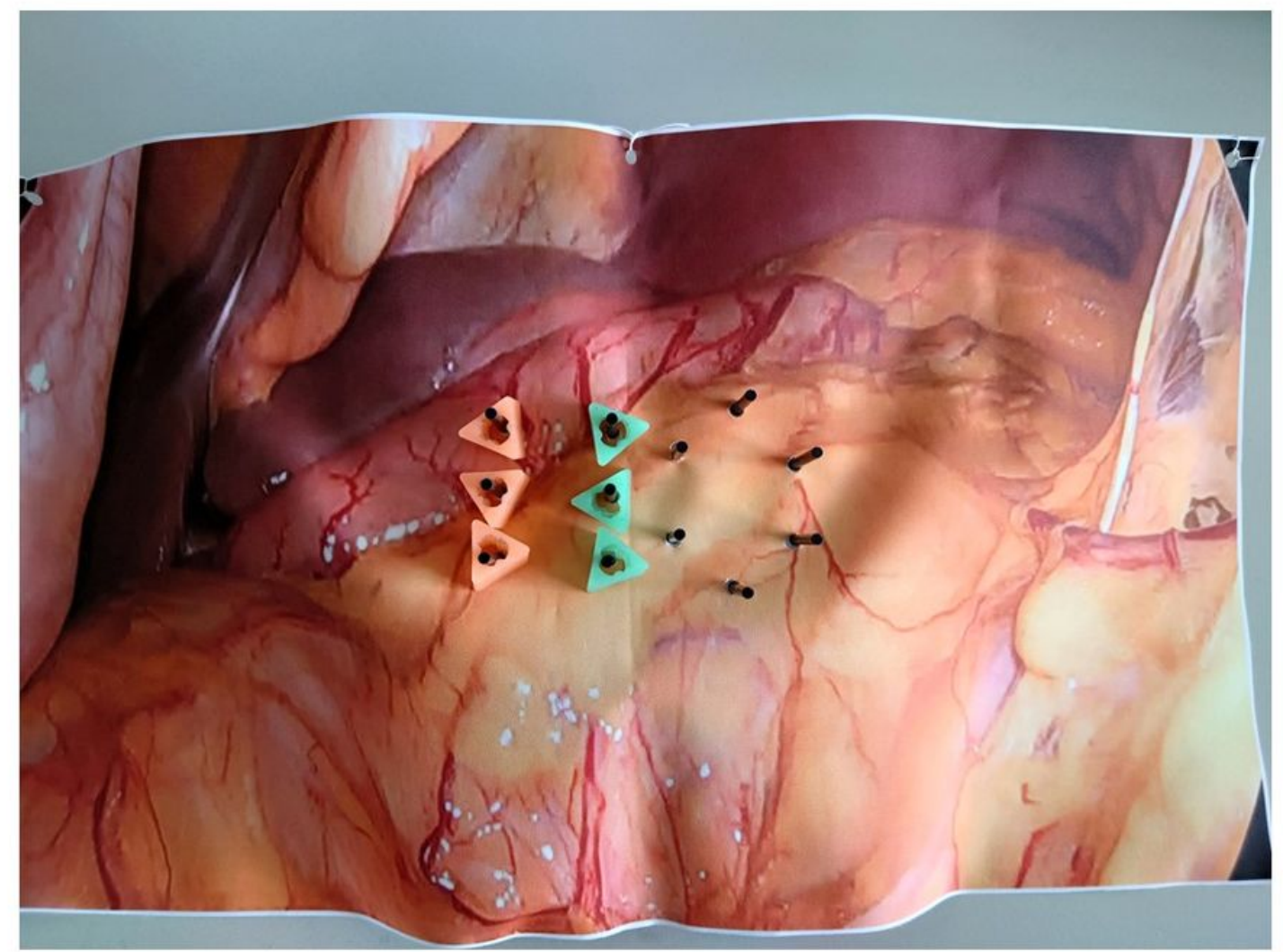

\section{Figure 1}

A peg board was placed on a table which mechanically rocked to mimic respiratory fluctuation. A cloth printed with intra-abdominal images was used as a background. 


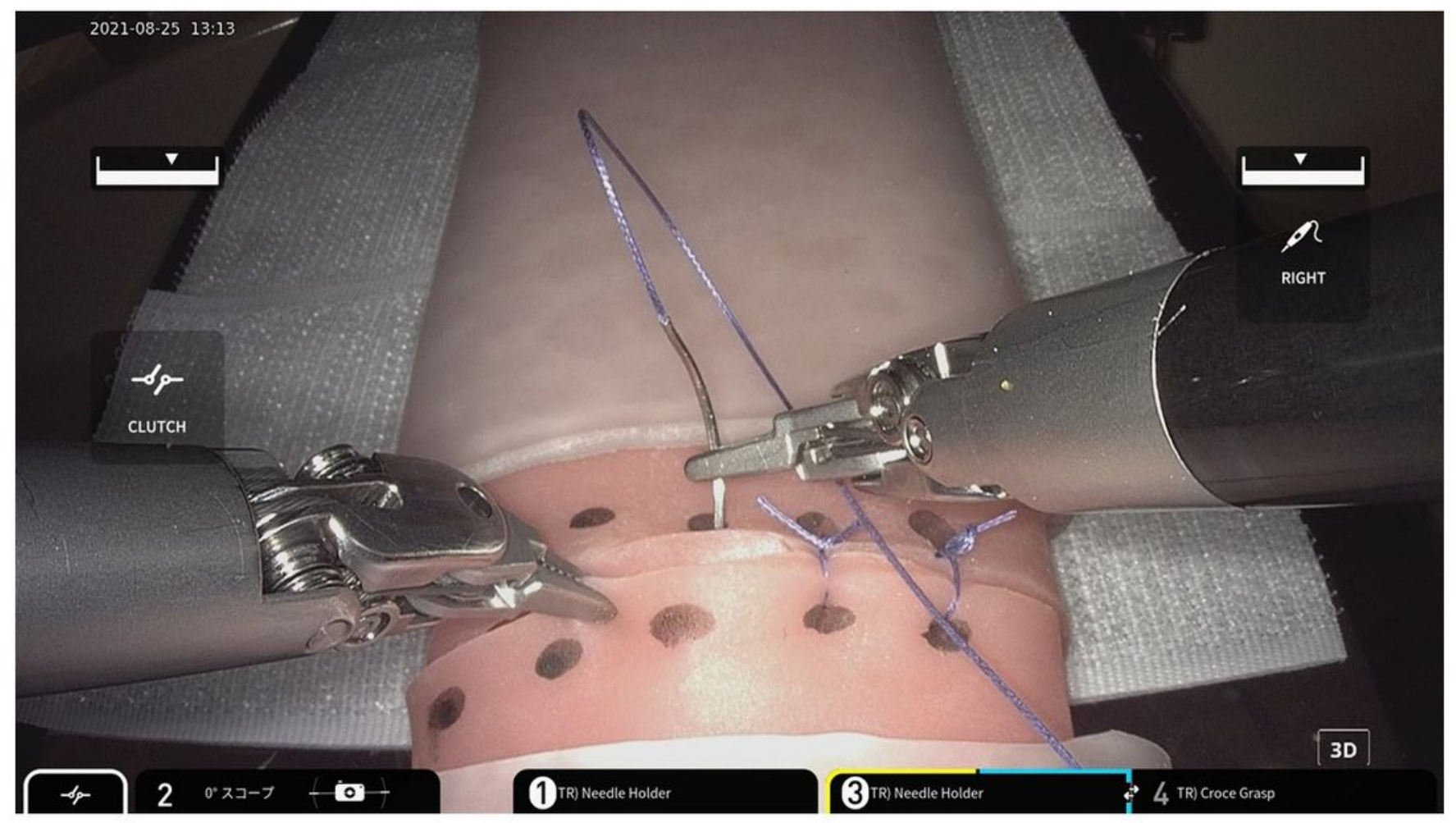

Figure 2

Five locations were marked at $5 \mathrm{~mm}$ intervals. Three single ligations were performed in one suture.

\section{Supplementary Files}

This is a list of supplementary files associated with this preprint. Click to download.

- SupplementaryTable.docx 